\title{
Mosaico Vítreo Retrorreflectante
}

\author{
A. BELDA ${ }^{1}$, Mª. ORTS ${ }^{1}$, F. VICIANO ${ }^{2}$ Y F. LUCAS ${ }^{2}$ \\ 1'-Instituto de Tecnología Cerámica, ITC. Campus Universitario Riu Sec, Avda. de Vicent Sos Baynat s/n. 12006 Castellón \\ 2. Salquisa S.A.. Polígono Industrial Gaido.12180 Cabanes. Castellón
}

Este trabajo ha participado en los premios Alfa de Oro en la Feria Internacional de Cerámica de Valencia CEVISAMA 2012

Salquisa y Alttoglass presentan un producto realmente innovador : mosaico vítreo retrorreflectante útil para su uso en demarcación vial horizontal y vertical, así como en arquitectura interior y exterior.

El producto presentado tiene muchas ventajas con respecto al sistema usado habitualmente. Se describen en la memoria los aspectos más diferenciadores. El aspecto fundamental es tener un producto de naturaleza exclusivamente vítrea y, por tanto, con unas características de durabilidad muy superiores a las pinturas, etc. Otras características le aportan también muchas ventajas descritas en la memoria.

Se consigue así abrir el uso del mosaico vítreo a la ingeniería civil y más concretamente a mejorar los aspectos de visibilidad nocturna tanto en condiciones std como en las condiciones más duras (lluvia por la noche, etc.). Mayor visibilidad = mayor seguridad.

Recordemos que el mayor índice de siniestralidad se produce en condiciones de lluvia nocturna.

La presentación del mosaico vítreo enmallado permite utilizarlo en paramentos verticales planos y curvos, tanto en señalización como en arquitectura interior y exterior.

El efecto de retrorreflexión permanece bajo el agua, por lo que también puede ser utilizado en fuentes ornamentales, piscinas etc.

El efecto puede conseguirse también sobre piezas cerámicas de gran formato.

El producto está protegido por la correspondiente PATENTE.

El proyecto se ha desarrollado con la colaboración del Instituto de Tecnología Cerámica, y cuenta con el apoyo del CDTI.

Palabras clave: Reflectante, mosaico, Visibilidad, señalización, vitreo

\section{Retro reflective glass mosaic}

Salquisa and Alttoglass have developed a very innovative product : the retro reflective glass mosaic. This new product can be used in both horizontal and vertical signposting and also in interior design and architecture.

This particular product has many advantages compare to the traditional methods used for signposting, design or architecture. One of them is that the product is mainly made of glass therefore it can last much longer than paints for example.

The used of glass mosaic for civil engineering it is opened up especially for signposting and it contributes to improve visibility at night not only in standard conditions but also in the hard ones such as wind, fog or rain at nighttimes. Higher visibility = higher security. We should remember that a high percentage of accidents occur under rain conditions at night.

The glass mosaic is presented in a mesh which allows the use in both plane and curve surfaces in signposting, interior design and architecture.

The retro reflective effect last under the water therefore the mosaic can be fixed in ornamental and decorative fountains, swimming pools, etc. Furthermore, the retro reflective effect can also be applied on big size ceramic tiles.

This project was developed along with the Institute of Ceramic Technology (ITC), it was supported by the Center for Industrial Technological Development (CDTI) and it is also patented.

Keywords: Reflective, mosaic, visibility, signposting, glass

\section{DESCRIPCIÓN DEL PRODUCTO}

El producto desarrollado por SALQUISA es un elemento de demarcación vial con propiedades retrorreflectantes constituido por mosaico vítreo de diversos colores, en cuya capa superior incluye microesferas de vidrio embebidas en su seno, que actúan como lentes esféricas retrorreflectantes, es decir, con capacidad para reflejar la luz incidente y devolverla hacia el observador en un ángulo muy parecido al de incidencia.

Las microesferas se han adherido al mosaico vítreo por una capa intermedia de un esmalte cerámico opaco, el cual ha reblandecido tras ser sometido a un proceso de cocción.
Con este reblandecimiento se ha producido la sinterización del esmalte y la adhesión de las microesferas de vidrio al conjunto: esmalte, mosaico vítreo.

La naturaleza del esmalte y las condiciones de cocción se han optimizado para conseguir la máxima adhesión de las microesferas, el \% de embebimiento adecuado de las mismas que garantizase la máxima retrorreflectividad (embebimiento entre el 55\% y 70\% del diámetro de la microesfera) y la máxima opacidad del esmalte, para que sea capaz de actuar como puntos de luz al tener la capacidad de difundir difusamente la luz que le llega a través de la microesfera. 
Las características técnicas (resistencia mecánica, resistencia química, etc.) se han controlado de forma comparativa a los productos std (pintura + microesferas), siendo el producto desarrollado muy superior en todos los ensayos.

El mosaico retrorreflectante se presenta enmallado al igual que otros mosaicos vítreos para así poder facilitar su colocación tanto en marcas viales, como en otros elementos retrorreflectivos: señales de demarcación vial horizontal, señales de demarcación vial vertical, otras señalizaciones, etc. También puede ser usado en arquitectura para recubrimientos en interior y también en exteriores.

El desarrollo presentado se basa en mosaico vítreo, pero puede hacerse también sobre piezas cerámicas, con el mismo proceso productivo.

\section{MÉTODO DE OBTENCIÓN DEL PRODUCTO}

La fabricación del mosaico vítreo retrorreflectante se realiza según las etapas siguientes:

1. Fabricación del mosaico vítreo convencional

2. Pulverización, en fase líquida, del esmalte opaco de naturaleza cerámica

3. Aplicación de las microesferas de vidrio cuando el esmalte está todavía húmedo para facilitar la penetración de las mismas en el seno del esmalte. Es muy importante regular la cantidad de esmalte vítreo, la adherencia del mismo al soporte, y la capacidad de adherencia a las microesferas para que el embebimiento de las microesferas sea el adecuado.

4. Secado.

5. Cocción de las piezas preparadas siguiendo un ciclo térmico específico: temperatura máxima inferior a la de reblandecimiento del mosaico vítreo y a la de las microesferas, pero adecuada para que el esmalte funda y sirva de anclaje.

6. Enmallado de las teselas de mosaico.

7. La aplicación de las bandas de mosaico sobre el asfalto se realiza por pegado con un adhesivo elástico utilizado para el pegado de los captafaros que se colocan en carreteras.

La fabricación puede hacerse también por monococción, partiendo de mosaico sin cocer, aplicando el esmalte en forma de granilla fina .

En la siguiente microfotografía podemos observar la superficie de las piezas obtenidas:

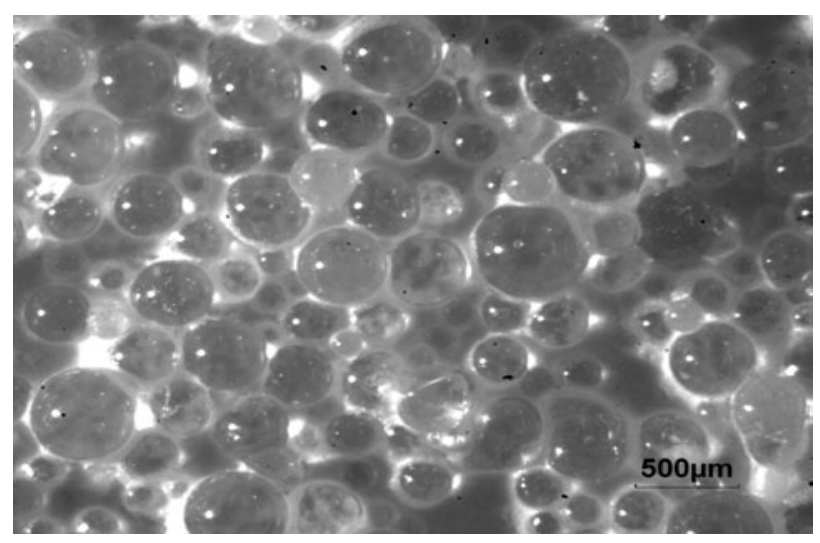

\section{CARACTERÍSTICAS ESPECÍFICAS DE ESTE PRODUCTO}

El mosaico vítreo descrito tiene unas características específicas que lo hacen diferente de los productos existentes hasta ahora, y que son:

- Todo el producto es de naturaleza vítrea y está sinterizado formando un solo cuerpo, por lo que no existen sistemas de pegado que puedan degradarse con el tiempo.

- El nivel de embebimiento de las microesferas en el esmalte está totalmente controlado durante la etapa de esmaltado y cocción, por lo que se obtiene un nivel de embebimiento de máximo rendimiento.

- La adherencia de las microesferas al esmalte y al mosaico es de naturaleza vítrea, con lo que se consigue un agarre muy superior al que se consigue con pinturas, adhesivos etc.: máxima duración incluso con tráfico rodado.

- El espesor del mosaico vítreo hace que, cuando de usa en señalización vial horizontal, quede unos $5 \mathrm{~mm}$. por encima del nivel del suelo, con una separación entre mosaico y mosaico de unos 2,5 mm., lo que permite un drenaje muy rápido en condiciones de lluvia, y se consigue mucha mayor visibilidad en estas condiciones .

- La presentación del producto enmallado igual que el mosaico vítreo tradicional lo hace perfectamente utilizable para usos de demarcación vertical, tanto en interiores como en exteriores, superficies planas y curvas, y con una gama de colores muy amplia. La presentación del producto enmallado y colocación en líneas produce también el efecto de ruido al rodar sobre él, avisando de que se está sobrepasando la línea de borde de la carretera (actualmente se colocan bandas sobre las líneas).

\section{USOS POSIBLES CON ESTE PRODUCTO}

- Señalización vial horizontal:

- Carreteras

- Aeropuertos

- Aparcamientos

- Pasos de peatones, ...

\section{- Señalización vial vertical:}

- Columnas y paredes en aparcamientos

- Zonas de baja visibilidad en carreteras

- Edificios, farolas, rotondas, ...

\section{- Arquitectura:}

- Interiores

- Exteriores: edificios singulares con iluminación externa, puentes, ...

- Piscinas o fuentes ornamentales: el efecto que se obtiene bajo el agua es muy diferente al de los recubrimientos habituales, consiguiéndose reflejos espectaculares.

$$
\text { - ... }
$$

\section{CARACTERÍSTICAS TÉCNICAS}

-Microesferas :

- Las microesferas utilizadas cumplen la normativa europea EN1423: 1997/A1: 
- Granulometría: 125 - 850 micras (Norma ISO 2591-1)

- Índice de refracción: Clase A (ind ref: 1.5)

- Resistencia química: Resistencia a: agua, ácido clorhídrico, cloruro de calcio, y sulfuro de sodio (anexo B de la Norma EN1423 : 1997/A1)

- Producto acabado (ensayo comparativo con el producto tradicional):

- Resistencia química según Norma ISO 10545 -13 ..... Clase : HA

- Resistencia al desgaste: realizado ensayo comparativo con la aplicación std (pintura especifica para señalización vial y microesferas) PEI según Norma EN - ISO 10545-7 evidencia mucho mejor resultado el producto desarrollado de naturaleza vítrea, llegándose en el producto std al arranque de la pintura a $600 \mathrm{rev}$.

- Ensayo de campo: realizado en las instalaciones de Salquisa, de forma comparativa con el producto tradicional:

El ensayo de campo está descrito en la Normativa (UNE 135200-3) y proporciona información sobre los efectos que produce en las marcas viales la interacción múltiple de diversos factores, difícilmente controlables que afectan al desgaste de las mismas.

- Producto y colocación tradicional:

- Limpieza de la superficie

- Pintura acrílica homologada: GRSF GR-201 Amarilla

- Microesferas de vidrio

- Nuevo producto:

- Limpieza de la superficie

- Colocación por pegado de las piezas de mosaico ya enmalladas

El ensayo de campo realizado hasta ahora (2 meses) evidencia, en situaciones de seco y en situaciones de lluvia un mejor comportamiento que el producto y colocación tradicionales, por agarre, mejor retrorreflexión en húmedo por el drenaje que realizan las teselas, y mayor duración del efecto ligado a una mayor adherencia de las microesferas al soporte.

Se nota también una mayor uniformidad en la superficie de retrorreflexión, al estar controladas tanto la densidad superficial de la microesferas como el embebimiento de las mismas en el soporte (difícilmente controlable sobre la pintura).

Colocación del producto en el aparcamiento de Salquisa:

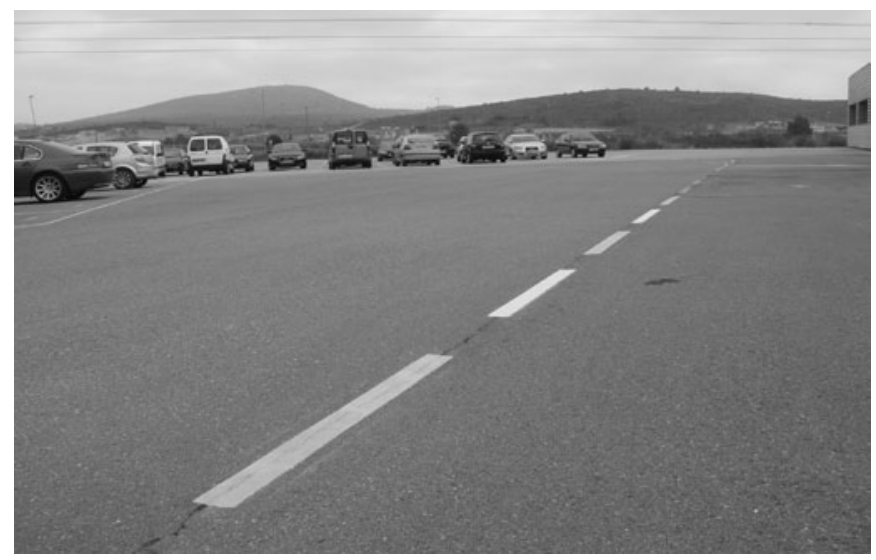

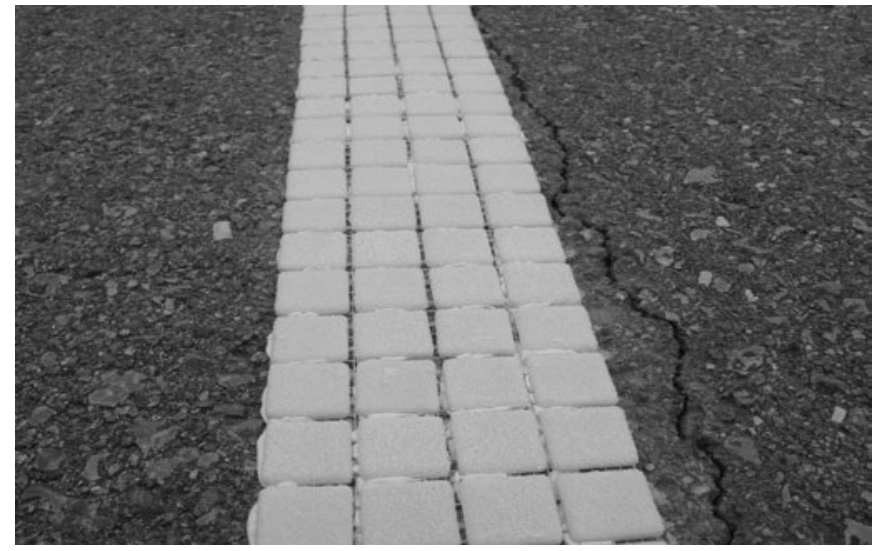

\section{INFORMACIÓN GENERAL SOBRE EL EFECTO DE LA RETRORREFLECTIVIDAD}

Se define la retrorreflexión como la luz que es reflejada por un elemento óptico y devuelta en la misma dirección (o casi) que la luz incidente procedente de una fuente de luz.

Esta propiedad se utiliza en demarcación vial, en la que se utilizan sistemas de marcado que aumentan la visibilidad nocturna, tanto en seco como en húmedo y además bajo condiciones de lluvia intensa.

Una marca vial está compuesta por una pintura que es responsable de la visibilidad diurna de la marca y que además actúa como ligante de un gran número de microesferas de vidrio embebidas en su seno, las cuales actúan como lentes esféricas con propiedades retrorreflectantes, por lo que son capaces de devolver una gran parte de la luz incidente procedente de una fuente de luz (los faros de un vehículo) en la misma dirección, y por lo tanto aumentan la visibilidad nocturna de la marca.

Las microesferas de vidrio presentan un índice de refracción y tamaño determinado, las cuales pueden estar incorporadas sobre las pinturas reflectantes directamente en su composición, o bien se espolvorean sobre la superficie recién pintada, antes de que la superficie se seque, para que queden parcialmente embebidas en la pintura. De este modo se consigue mejorar el efecto reflectante de la pintura, con respecto a que llevan las esferas de vidrio en composición.

\subsection{Factores que afectan a la eficacia de la retrorreflectividad}

\section{Análisis de la retrorreflectividad}

Para analizar los factores de los que depende la retrorreflectividad es necesario realizar previamente un análisis de la situación.

Si se asume que la distancia a la que un conductor de automóvil en movimiento visualiza las marcas viales, varía entre $12 \mathrm{~m}$ (mínima distancia perceptible) y $122 \mathrm{~m}$ (máxima distancia), y teniendo en cuenta que la altura media de los faros de un automóvil sobre la calzada es de $61 \mathrm{~cm}$, el ángulo de inclinación de la luz sobre la marca varía entre $2,7^{\circ}$ y $0,3^{\circ}$. Esta luz incidente sobre la superficie del sistema óptico es parcialmente reflejada hacia delante, parcialmente absorbida por la propia superficie de la carretera; y sólo una parte de la luz será retrorreflejada y devuelta hacia los ojos del conductor, 
por las microesferas de vidrio que contiene la marca vial. Estableciendo las consideraciones geométricas oportunas se obtiene que el intervalo de ángulos de retrorreflexión útil está comprendido entre $0^{\circ}$ y $3^{\circ}(1)$.

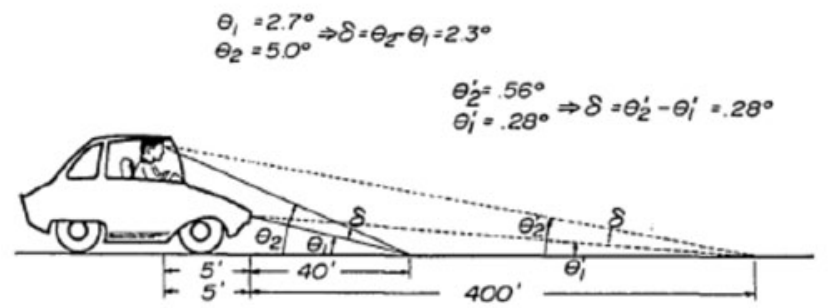

Figura 1.- Condiciones aproximadas de ángulos de incidencia y de retrorreflexión en marcas de pavimento horizontal

Se define el nivel de "embebido" o de inmersión de las microesferas en la pintura como el porcentaje del diámetro de la esfera que esta cubierto por la pintura, y se asume siempre que dicho nivel es horizontal. Las microesferas que están embebidas en la pintura menos del $50 \%$ de su diámetro no son útiles para la retrorreflexión porque tienen una probabilidad elevada de ser arrancadas por el uso.

Cuando la luz procedente de los faros de un automóvil incide sobre las esferas de vidrio que están parcialmente embebidas en una pintura, parte se pierde por reflexión especular desde la superficie lisa de la esfera, mientras que el resto se refracta hacia la parte trasera de la esfera. Cuando la luz llegue a la parte trasera de la esfera sufrirá una nueva reflexión especular interna y el resto de luz será refractada hacia la parte exterior de la esfera. Pero si la esfera está pintada en el punto de emergencia, esta luz será difusamente dispersa en todas las direcciones.

Teóricamente es posible obtener retrorreflexión útil usando esferas de vidrio de alto índice de refracción operando en forma de reflexión especular interna simple. Sin embargo, como las microesferas de vidrio usadas en demarcación horizontal están embebidas al menos el $50 \%$ de su diámetro (para garantizar la estabilidad mecánica de la marca), se puede demostrar que los rayos retrorreflejados por reflexión interna especular se encontrarán la superficie frontal de salida de la esfera por debajo del nivel de embebido, por lo que nunca saldrán al exterior. El rayo que consiga salir, el que esté próximo a la interfacie vidrio-aire será capaz de salir de la esfera, pero saldrá con un ángulo con respecto a la normal demasiado grande para ser luego aprovechado por el

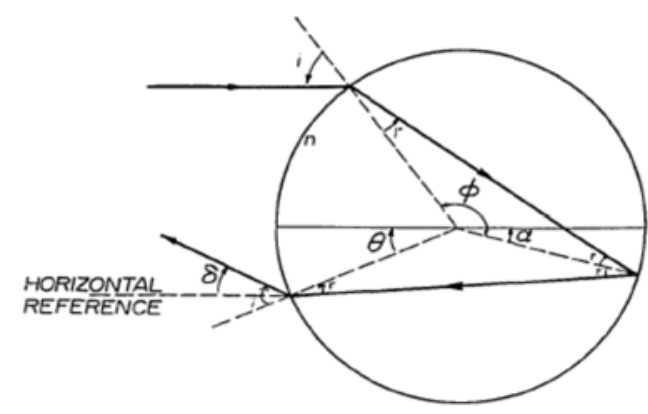

Figura 2.- Diagrama que sigue un rayo de luz incidente en una esfera de vidrio. Caso de reflexión especular en la parte de atrás de la esfera. conductor del automóvil, es decir, no estará comprendido en el intervalo útil de ángulos de retrorreflexión (ver Figura 2).

En el caso de múltiples reflexiones internas, se pueden calcular los ángulos de salida de la luz retrorreflejada de la esfera, para esferas de distinto índice de refracción y en función del número de reflexiones internas, y si además tenemos en cuenta que sólo los ángulos de salida entre 0 y $3^{\circ}$ son aprovechables, se puede demostrar que los ángulos posibles son siempre inferiores al ángulo crítico, por lo que ninguna reflexión interna conseguirá salir de la esfera.

En consecuencia, la reflexión especular interna no contribuye a la intensidad de la luz retrorreflejada que es aprovechable por el conductor.

Para analizar la contribución a la retrorreflexión de la reflexión difusa (3) es necesario primero considerar la sección vertical de una esfera que está por encima del nivel de embebido de la superficie, a la que le llega un rayo de luz incidente con dirección paralela a la horizontal (ver Figura 3). Si $\alpha$ es el ángulo entre el diámetro horizontal y la línea que une el centro de la esfera con el punto de la esfera en el que se produce la refracción interior. Toda la luz que incida en la parte frontal de la esfera será refractada dentro de un casquete esférico cuyo radio dependerá del seno de dicho ángulo. Se puede demostrar aplicando consideraciones geométricas y la ley de Snell de la refracción, que este ángulo depende del ángulo de incidencia y del índice de refracción de la esfera y que para un determinado índice de refracción aumenta ligeramente cuando aumenta el ángulo de incidencia y a partir de un cierto valor empieza a disminuir, por lo que la distribución de la intensidad o la densidad de flujo de la luz refractada no será uniforme en dicho casquete esférico.

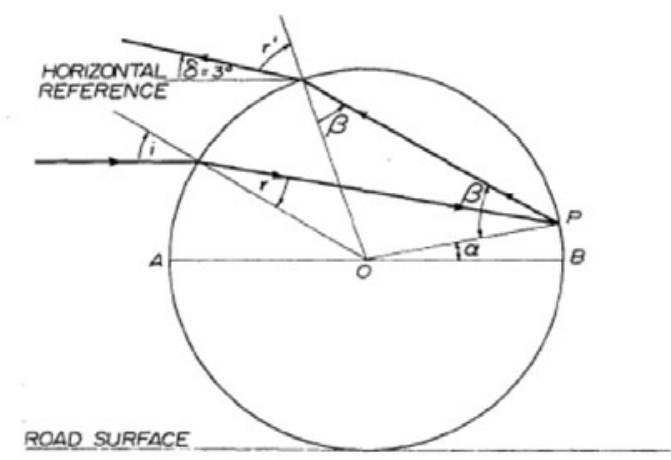

Figura 3.- Diagrama que sigue un rayo de luz incidente en una esfera de vidrio.

Si la esfera no estuviese embebida en la pintura, la luz emergería por la parte de atrás de la esfera por una nueva refracción y se perdería. Sin embargo al estar pintada la parte trasera de la esfera, cada punto del casquete esférico actuará como un punto fuente de luz y esta luz será difusamente reflejada desde cada punto. Conforme se incrementa el nivel de embebido de las microesferas el casquete esférico actúa más y la reflexión difusa contribuye con mayor intensidad a la luz retrorreflejada, siempre y cuando dicho casquete esférico no se encuentre por debajo del nivel de embebido. En consecuencia, existe un nivel óptimo de embebido de las microesferas de vidrio en la pintura que depende del índice de refracción de las mismas. 
En la figura 4 se detalla la sección vertical de una microesfera de vidrio embebida arbitrariamente en una pintura. El rayo de luz incidente en la esfera con un ángulo i, se encuentra la superficie interna de la esfera en el punto A (por debajo del nivel de embebido) y genera un punto de luz. La luz difusamente reflejada en ese punto se divide en tres categorías:

1. Las líneas de puntos representan los rayos de luz reflejados directamente a partir de la luz incidente, los cuales o bien llegarán a la parte frontal de la esfera por debajo del nivel de embebido, por lo que se perderán para el observador, o bien saldrán de la esfera con unos ángulos tan pequeños que no serán capaces de llegar al observador.

2. Las líneas continuas por encima del nivel de embebido representan los rayos de luz difusamente reflejados. Los rayos para los que $\mathrm{r}<\beta<\beta_{\text {crit' }}$ el ángulo de retroreflexión $\delta$ será mayor que cero, y proporcionan luz útil como luz retrorreflejada. Los rayos para los que $\beta>\beta_{\text {crit }}$ serán reflejados totalmente internamente, por lo que no saldrán de la esfera.

3. Las líneas a trazos los rayos reflejados difusamente para los que $\beta>\beta_{\text {crit' }}$ por lo que como ya se ha comentado anteriormente, no emergerán de la esfera y se perderán para el observador.

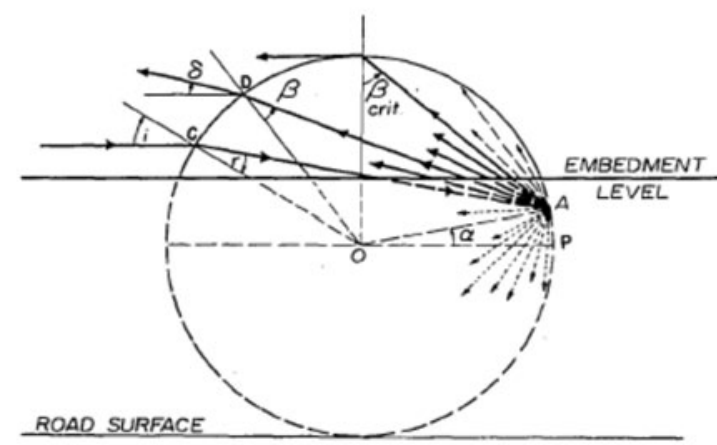

Figura 4.- Diagrama que sigue la luz incidente en una esfera de vidrio. Caso de refracción difusa.

Es decir, sólo los rayos de luz reflejados difusamente y que satisfagan el criterio de $\mathrm{r}<\boldsymbol{\beta}<\beta_{\text {crit }}$ pueden proporcionar luz útil y aprovechable para un conductor de automóvil. Existiendo además algunas limitaciones adicionales, como son el índice de refracción de las microesferas de vidrio y la posición del punto A, posición que a su vez depende del nivel de embebido de las microesferas. Además todas las consideraciones geométricas se han realizado para lentes esféricas por lo que la esfericidad de la microesferas de vidrio ha ser máxima.

\section{Factores que influyen en la Eficacia de la Retrorreflexión}

Para estudiar la Eficacia en la Retrorreflexión de las microesferas de vidrio es necesario asumir que la intensidad de luz incidente se mantiene constante.

La eficacia en la retrorreflexión de la luz disminuye de forma muy acusada con la distancia, para cualquier índice de refracción de las microesferas y para todos los niveles de embebido (ver figura 5). El aumento del índice de refracción de

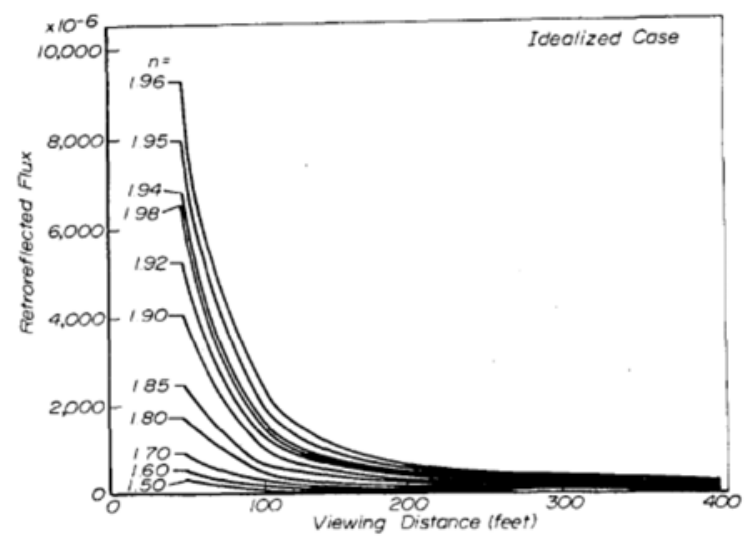

Figura 5.- Variación del Flujo de luz retrorreflejada con la distancia para esferas de distinto índice de refracción.

las microesferas incrementa considerablemente la cantidad de luz retrorreflejada, aunque por encima de índice de refracción 1,96 el flujo de luz retrorreflejada disminuye porque la región de casquete esférico se reduce gradualmente a un punto.

En la Figura 6 se detalla la variación del Flujo de luz retrorreflejada en función del grado de embebido para varias distancias de iluminación.

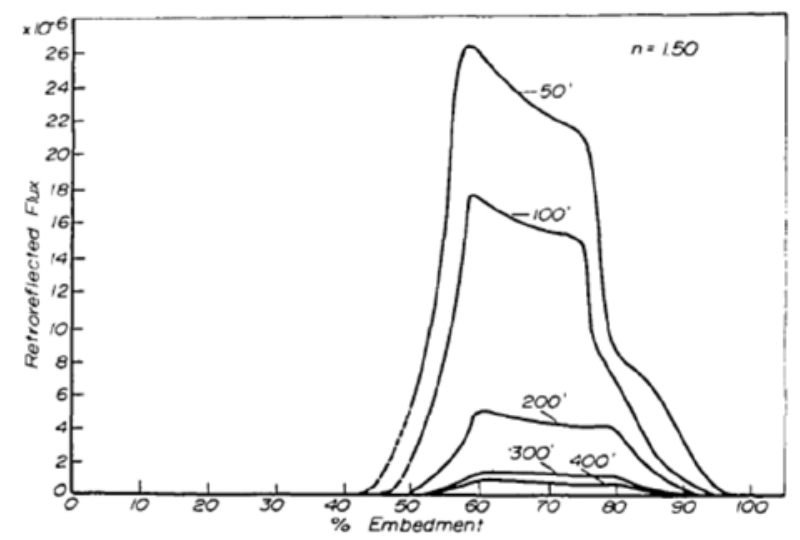

Figura 6.- Variación del Flujo de luz retrorreflejada para una esfera de índice de refracción $\mathrm{n}=1,5$ y para distintos valores de nivel de embebido.

De la observación de la figura 6 se deduce que la luz retrorreflejada es despreciable para cualquier nivel de embebido de las esferas inferior al 50\% del diámetro de la esfera y también para niveles de embebido superior al $90 \%$. Además, para un determinado grado de embebido la luz retrorreflejada desciende rápidamente al aumentar la distancia de iluminación y observación. Además existe un grado de embebido para cada distancia, al cual el nivel de retrorreflexión es máximo, el cual para esferas de índice de refracción de 1,5 es el 59\% del diámetro de la esfera.

Figuras similares pueden derivarse para esferas de otros índices de refracción, pero la conclusión final que se obtendría sería la misma: La mayor eficacia en el retrorreflexión de marcas de pavimento se obtiene cuando se eligen esferas de 
vidrio con índice de refracción lo más cercano a 2, aunque hay que tener en consideración otros factores como económico, de resistencia mecánica, durabilidad y disponibilidad, etc.

\section{Propiedades ópticas bajo condiciones de humedad y lluvia}

La eficacia de la retrorreflexión disminuye notablemente bajo condiciones de humedad y lluvia, debido a que cuando llueve, el agua moja la superficie de las marcas de pavimento y se acumula formando una capa continua de agua en la superficie de la marca (4). El espesor de dicha capa por encima de las microesferas depende de la intensidad de la lluvia y del drenado de la marca vial. El agua tiene un índice de refracción superior al del agua (1,3 del agua frente a 1 del aire), por lo que cuando incide un rayo de luz sobre la microesfera (ver Figura 7), la primera refracción de la luz incidente se produce al pasar al agua, y dependiendo del espesor de la capa de agua, y de si esta capa adopta curvatura alrededor de las lentes, el camino óptico que seguirá el rayo de luz será el que se muestra en Figura 7 (b) ó en la 7(c).

En estas condiciones las lentes de índice de refracción de 1,5 actúan como si se tratase de 1,1 en el aire, y las lentes más efectivas en el aire, cuyo índice de refracción es de 1,9 se convierten en 1,5. Para obtener un índice de refracción funcional de 1,9 en condiciones de lluvia continua se necesitan lentes esféricas de índice de refracción del orden de 2,5. La máxima retrorreflectividad bajo condiciones de lluvia continua requiere el uso de sistemas de marcado que incluyan este tipo de lentes de elevado índice de refracción.

\subsection{Otras informaciones de interés :}

Los elementos retrorreflexivos que contienen las microesferas se describen en la Patente US 2007/0110960 A1, entre las que se incluyen las marcas viales.

En los elementos reflectivos de uso en señales, aparatos, etc., el ligante que fija las microesferas normalmente es transparente. Los ligantes transparentes se aplican sobre una base reflectiva o puede ser aplicado sobre un soporte sobre el que se aplicarían las microesferas y una vez solidificado el ligante, se podría despegar para obtener un film reflectante que se pueda luego pegar en otras superficies (tipo una "pegatina").

Se describe un núcleo central que puede ser un material inorgánico, tipo vitrocerámico en el que la fase cristalina actúa para dispersar la luz resultando una apariencia opaca o semitransparente, o bien un material orgánico tipo un termoplástico o una resina, por ejemplo epoxi, poliuretano, alquilo, acrílico, poliésteres y fenólicos. Materiales epóxidos, poliuretanos y poliésteres se describen en las patentes U.S. $\mathrm{N}^{\mathrm{o}}$ $3,254,563 ; 3,418,896$ y $3,272,827$

\section{CONCLUSIONES}

- Los elementos retrorreflexivos que son objeto del presente desarrollo están compuestos de un material inorgánico de naturaleza exclusivamente vítrea (mosaico vítreo obtenido según procedimiento descrito).

- No se describe en la bibliografía ningún elemento retrorreflexivo obtenido con material de naturaleza exclusivamente vítrea.

- En la patente también se reivindica el ligante, el cual es un material inorgánico de naturaleza cerámica ( esmalte vítreo) y el procedimiento de obtención del esmalte y del elemento retrorreflexivo.

- Se abren así nuevos horizontes para el mosaico vítreo, con unos usos de alto valor añadido, y un potencial altísimo por cubrir unas necesidades no resueltas hasta ahora.

- El producto presentado tiene muchas ventajas con respecto al sistema usado hasta ahora (pintura + microesferas) y permite ser utilizado en paramentos verticales (planos y curvos) tanto en señalización como en arquitectura interior y exterior

- El efecto se consigue también sobre piezas cerámicas de gran formato, siendo preferible (aunque no excluyente) el uso de piezas de gres porcelánico (se presentan también en Cevisama).

\section{BIBLIOGRAFÍA}

La bibliografía más relevante consultada, además de las patentes y normas referidas en el documento, es:

(1) Stoudt, M.D.; Vedam, K.: Retrorreflection from spherical glass beads in highway pavement markings. 1: Specular reflection. Applied Optics, Vol. 17, No 12, p. 1855. 1978.

(2) Stoudt, M.D.; Vedam, K.: Retrorreflection from spherical glass beads in highway pavement markings. 2: Diffuse reflection (a first approximation calculation). Applied Optics, Vol. 17, No 12, p. 1859. 1978.

(3) Burns, D.M.; Hedblom and Miller: Modern pavement marking systems: The relationship between optics and nighttime visibility. 87 th Annual Meeting of Transportation Research Board. January 13-17, 2008.

(4) Pavement marking, reflective elements, and Methods of making microspheres. Pat US 2007/0110960 A1. Solicitante 3M Innovative Properties Company.

(5) Pavement marking and reflective elements having microspheres comprising Lanthanum oxide and aluminium oxide with zirconia or, mixures thereof. Pat WO 2008/140864 A1. Solicitante 3M Innovative Properties Company

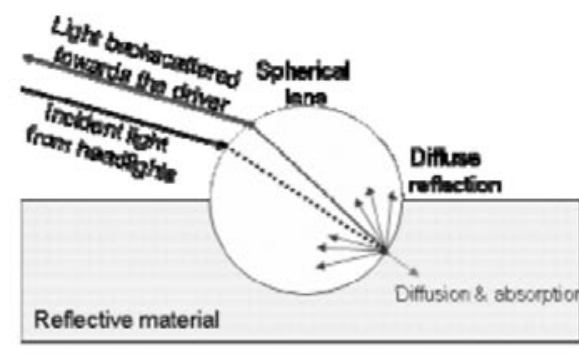

(a)

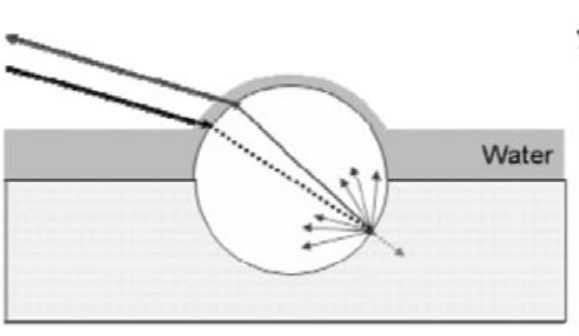

(b)

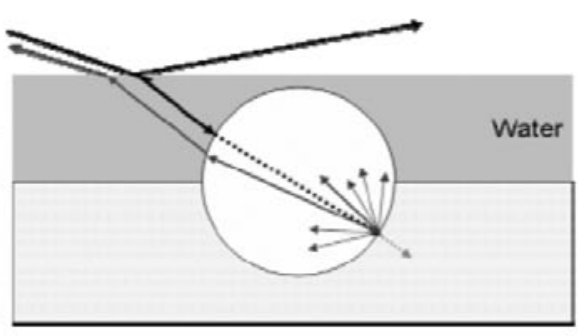

(c)

Figura 7.- Variación del camino óptico de la luz bajo diferentes condiciones. (a) Sistema óptico seco. (b) Sistema óptico húmedo. (c) Sistema óptico mojado. 